\title{
Design and Improvement of Routing Protocol for Field Observation Instrument Networking Based on LEACH Protocol
}

\author{
Jiuyuan Huo (iD, Xingyue Deng, and Hamzah Murad Mohammed Al-Neshmi \\ School of Electronic and Information Engineering, Lanzhou Jiaotong University, Lanzhou 730070, China \\ Correspondence should be addressed to Jiuyuan Huo; huojy@foxmail.com
}

Received 3 May 2020; Accepted 13 August 2020; Published 1 September 2020

Academic Editor: José Luis Domínguez-García

Copyright (C) 2020 Jiuyuan Huo et al. This is an open access article distributed under the Creative Commons Attribution License, which permits unrestricted use, distribution, and reproduction in any medium, provided the original work is properly cited.

\begin{abstract}
Field observation instruments in cold and arid areas are deployed with many difficulties caused by the harsh natural environment, which leads to the lag of information acquisition ability and severely restricts the geoscience research in these areas. Therefore, it is urgent to study the suitable routing technology of observation instrument networks according to the characteristics of cold and arid areas. In this paper, we have studied and designed an improved routing protocol for the field observation instruments network based on the LEACH protocol (FOI-LEACH). Firstly, the FOI-LEACH was proposed to mainly improve the LEACH protocol in three aspects: (1) the network nodes are heterogeneous and combined with the characteristics of field observation instrument networking. The residual energy and the rechargeable energy of nodes are added in the process of cluster head $(\mathrm{CH})$ election to reduce the risk of premature death of $\mathrm{CHs}$ and shortened network life cycle caused by the selection of nodes with less energy as $\mathrm{CH}$. (2) In the process of cluster forming, the distance from $\mathrm{CH}$ to the base station (BS) and the residual energy of $\mathrm{CH}$ is considered when setting the cluster radius, to reasonably plan the cluster size and alleviate the "hot spot" problem. The nonuniform distribution of clusters in the network is enhanced to balance the total network energy consumption. (3) The autonomous zone-based multihop routing mechanism is adopted to solve the low reliability of data transmission caused by the poor quality of intercluster communication links and premature death of nodes in long-distance transmission. Then, MATLAB was used to compare the network routing protocol model of the observation instrument from four aspects, network life, energy consumption rate, stability, and throughput. The results showed that the improved algorithm FOI-LEACH balances the network energy consumption and alleviates the "hot spot" problem, to extend the lifetime of network nodes.
\end{abstract}

\section{Introduction}

The cold and arid areas in China represent more than twothirds of the country. The ecological environment in cold and arid areas is fragile but contains indispensable resources of the national economy and has a prominent strategic position [1]. Moreover, these areas have formed a field monitoring network system, composed of three-level positioning observation and research stations at the national, hospital, and institute levels, supplemented by other semipositioning observations and covering the main ecological environment areas in the cold and arid areas of our country. Through observation, experiment, and demonstration, the field monitoring network system provides an essential scientific basis for scientific research in such areas. It has become an indispensable and irreplaceable research force and support platform for scientific research in these areas. However, the field stations of cold and arid areas are located in the harsh natural environment, which leads to the lag of information acquisition ability and severely restricts the geological research in these areas [2]. Therefore, it is urgent to study the suitable routing technology for observation instrument networks according to the characteristics of these areas.

To establish the field observation instrument networks in cold and arid areas and promote full automation and realtime transmission of field observation data, we designed an instrument network node and gateway node [1]. The observation instrument network is mostly deployed in a harsh environment. Although the limited energy of nodes is improved by an auxiliary power supply of solar cells, the harsh environment leads to the problem of insufficient battery 
charging and the demand of uninterrupted data transmission. Hence, it is necessary to design a networking routing protocol with energy supply.

It is found that there are certain similarities between the investigation and analysis of the field observation environment in cold and arid areas on the one hand and the comparative analysis of wireless sensor network (WSN) [3] with highly integrated knowledge and well-advanced technology on the other hand, which are self-organizing network, dynamic topology, wireless transmission medium, and multihop network. Therefore, according to the characteristics of field observation instrument networking and WSN, the routing protocol is improved and optimized.

In WSNs, the Low Energy Adaptive Clustering Hierarchy (LEACH) [4] designed by Heinzehnan et al. of Massachusetts Institute of Technology in the USA is a classical and effective energy routing protocol based on clustering. As shown in Figure 1, the LEACH protocol belongs to the hierarchical routing protocols [5], which clusters nodes in the network, and reduces the amount of sent information by using data fusion technology. The sensor node does not need to maintain the routing table with comprehensive information, which can effectively improve the energy utilization of nodes and extend the average working time of the network. At the same time, the hierarchical structure improves the scalability of the WSN.

Networks are usually deployed in open, unattended environments, which make them more vulnerable to attacks. Therefore, it is crucial to devise security solutions to these networks. But LEACH is more robust against insider attacks than most other routing protocols [6]. In contrast to more conventional multihop schemes where nodes around the BS are especially attractive for compromise (because they concentrate all network-to-BS communication flows), $\mathrm{CHs}$ in LEACH communicate directly with the BS, can be anywhere in the network, and change from round to round. All these characteristics make it harder for an adversary to identify and compromise strategically more important nodes [7].

However, the LEACH algorithm still faces many challenges. For example, without considering the residual energy of nodes and the distance to the base station (BS), the randomness of cluster head election may cause the far away from the BS cluster head to die quickly due to excessive longdistance communication energy consumption, which affects the survival time of the whole network. In order to solve these problems in the LEACH protocol, FOI-LEACH, an improved algorithm for network energy balance, was proposed considering the energy supply. The algorithm was mainly improved from the following three aspects:

(1) The network nodes are heterogeneous and combined with the characteristics of field observation instrument networking. The residual energy and the rechargeable energy of nodes are added in the process of $\mathrm{CH}$ election to reduce the risk of premature death of $\mathrm{CHs}$ and shortened the network life cycle caused by the selection of nodes with less energy as $\mathrm{CH}$.

(2) In the process of cluster forming, the distance from $\mathrm{CH}$ to the $\mathrm{BS}$ and the residual energy of $\mathrm{CH}$ is

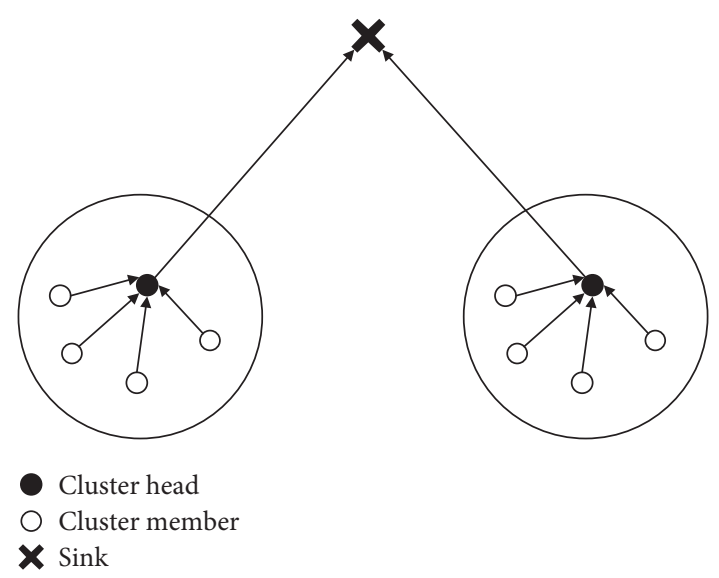

Figure 1: The hierarchical model of the LEACH protocol.

considered when setting the cluster radius, to reasonably plan the cluster size and alleviate the "hot spot" problem. The nonuniform distribution of clusters in the network is enhanced to balance the total network energy consumption.

(3) The autonomous zone-based multihop routing mechanism [8] is adopted to solve the low reliability of data transmission caused by the poor quality of intercluster communication links and premature death of nodes in long-distance transmission.

The paper is organized as follows: the related work was briefly described and reviewed in Section 2; in Section 3, the proposed FOI-LEACH protocol is described in detail; the network model of field observation instruments is established and analyzed in Section 4; the experiment settings, results, and corresponding analyses were discussed in Section 5; and finally, the conclusions are presented in Section 6 .

\section{Related Works}

LEACH routing protocol is a WSN routing algorithm designed by Heinzehnan et al. from MIT in the United States, which is the earliest typical hierarchical routing protocol [9]. LEACH protocol adopts the method of distributed $\mathrm{CH}$ election, in which some nodes are randomly selected from the network as $\mathrm{CHs}$, and other nodes become cluster member nodes [10]. The $\mathrm{CH}$ broadcasts the message that it becomes a $\mathrm{CH}$, and other nodes select the $\mathrm{CH}$ with the strongest received signal to join to form a cluster [9]. The cluster member node collects data and transmits it to the $\mathrm{CH}$, which receives data and transmits it to the BS through single-hop communication. The $\mathrm{CHs}$ undertake the heavy tasks, including managing the member nodes of the cluster, collecting the data transmitted by the member nodes, data fusion, and intercluster forwarding. Therefore, to balance the energy consumption of nodes, CHs rotate, and the cluster structure is updated periodically.

The basic idea of the LEACH protocol is to divide the network into clusters of equal size. The $\mathrm{CH}$ rotates periodically, and each cycle is called a "round." Each round is 
divided into two stages: the establishment stage of the cluster and the stable transmission stage [10].

In the establishment stage of the cluster, each node generates a random number from 0 to 1 , and the threshold $T(n)$ is calculated according to equation (1). Then, the random number generated by each node is compared with $T(n)$. If the value is less than $T(n)$, the node is selected as the $\mathrm{CH}$ :

$$
T(n)= \begin{cases}\frac{p}{1-p *(r \bmod ((1 / p)))}, & n \in G, \\ 0, & n \notin G,\end{cases}
$$

where $p$ is the percentage of $\mathrm{CH}$ in all nodes, $r$ is the number of current election rounds, $r \bmod (1 / p)$ is the number of nodes that have been selected in this round, and $G$ is the set of nodes without $\mathrm{CHs}$ selected in this round. After the end of each $\mathrm{CH}$ selection round, each selected $\mathrm{CH}$ broadcasts its message of becoming a $\mathrm{CH}$ to other nodes. After receiving the broadcast message, other nodes choose to join a cluster according to the received signal strength and send their joining message to the selected $\mathrm{CH}$ [11]. Each $\mathrm{CH}$ creates and assigns a TDMA schedule between each member node after its member nodes are joined. Then, end the cluster establishment stage and start the data transmission stage.

In the data transmission stage, each member node sends data to the $\mathrm{CH}$ within its allocated period, and the $\mathrm{CH}$ transmits data to the BS after data fusion. Therefore, $\mathrm{CHs}$ consume more energy than member nodes. LEACH ensures that all nodes are equally likely to act as $\mathrm{CHs}$ employing cycle circulation so that the nodes consume energy in a relatively balanced manner. However, factors such as residual energy of nodes and distance from the BS are still not considered. The randomness of the $\mathrm{CH}$ election may lead to the death of the $\mathrm{CH}$ far away from the BS due to the rapid exhaustion of energy, which affects the survival time of the whole network.

To address these issues, the researchers have proposed many improvements to the LEACH protocol for each stage. However, because of the difficulty, high cost, and timeconsuming problems of deploying nodes in the field, the researchers use MATLAB, NS2, OMNeT++, OPNET, and other simulation software to simulate the network, which can provide objective and reliable data for network planning and design, shorten the network simulation time, and reduce the investment cost. In this paper, we will use the MATLAB simulation platform to test and evaluate experimental performance. Heinzelman et al. proposed a centralized LEACH (C-LEACH) to improve the LEACH protocol performance. Instead of sensor nodes themselves, the sink selects $\mathrm{CHs}$ in C-LEACH and the algorithm gives better results than the LEACH algorithm [12]. In the case of $\mathrm{CH}$ election, one improvement scheme is to improve the threshold $T(n)$. For example, Sara al-sodairi considered the current residual energy of nodes to reduce the possibility that nodes with less energy are selected as $\mathrm{CHs}$ [13]. However, as the network runs for a long time, all the surviving nodes will have less energy. The corresponding threshold $T(n)$ also becomes smaller; that is, the probability of ordinary nodes being selected as $\mathrm{CHs}$ becomes smaller, and the number of $\mathrm{CHs}$ selected in each round decreases successively, which leads to unbalanced network energy consumption and shorter network survival time. Lots of researchers put forward methods for the cluster head election by considering more factors. In the hybrid energyefficient distributed (HEED) protocol, cluster heads are selected based on the nodes' remaining energy and node degree [14]. HEED can asymptotically almost surely guarantee connectivity of clustered networks, and simulation results demonstrated that it is effective in prolonging the network lifetime and supporting scalable data aggregation. Binbing Chen considered the current residual energy of the node and considered the current average energy of the network [15]. As the node's current energy value is less than the average energy value, it cannot be selected as the $\mathrm{CH}$. Compared with the original LEACH protocol, better quality nodes can be selected as $\mathrm{CHs}$, which can improve network performance and prolong the network life cycle to some extent. However, it does not prevent the $\mathrm{CH}$ from consuming more energy due to the heavy load and long-distance communication. In [16], fuzzy multiple attribute decisionmaking (MADM) approach is used to select $\mathrm{CHs}$ using three criteria including residual energy, number of neighbors, and the distance from the base station of the nodes. The simulation results demonstrated that it is more effective in prolonging the network lifetime in homogeneous environments. Soro and Heinzelman proposed cluster head election techniques for coverage preservation in WSN based on a set of coverage-aware cost metrics that favor nodes deployed in densely populated network areas as better candidates for cluster head nodes, active sensor nodes, and routers [17]. Compared with the traditional energy-based selection methods, the methods can increase the clustered sensor network lifetime. Thakkar took into account the factors such as residual energy, the mean value, and standard deviation of the current network energy and the distance from the node to the BS [18]. The added energy factor and distance factor can make the network energy change relatively stable, and the nodes close to the BS are more likely to be selected as $\mathrm{CHs}$, so the energy consumption of $\mathrm{CHs}$ is relatively balanced. Compared with the original LEACH, the protocol has a longer stable period and enhances the network stability. However, due to the randomness of the clusters generated in each round, the maximum and minimum clusters may be formed, making the distribution of network energy consumption unreasonable and shortening the network life cycle. In [19], Chang et al. solved the possibility of minimum clusters and maximum clusters. In the clustering stage, the node selects the $\mathrm{CH}$ according to the received broadcast signal strength and adds a parameter based on the clustering. The parameter comprehensively takes into account the distance between the node to each $\mathrm{CH}$ and the energy factors of the $\mathrm{CH}$. The algorithm can optimize the clustering process to reduce the occurrence of extreme cases such as maximum and minimum clusters and form a more reasonable cluster. It makes the distribution of energy consumption more reasonable and prolongs the life cycle of the network. Mehra et al. proposed the SE-LEACH protocol in [20], which 
considers the node density, residual energy, distance from the $\mathrm{BS}$, and power consumption in selecting the $\mathrm{CH}$. At the same time, the non- $\mathrm{CH}$ selects the $\mathrm{CH}$ according to the residual energy, node density, the power dissipated by the $\mathrm{CH}$ in the process of operation, and the distance to the $\mathrm{CH}$. This protocol can increase the stable area of WSN, balance the load, and ensure that all nodes similarly consume power.

Some scholars have also proposed routing protocols for heterogeneous network structures, Smaragdakis et al. proposed a stable heterogeneous election protocol SEP [21]. In the SEP protocol, two kinds of nodes with different initial energy are proposed, which are the normal node with less initial energy and the advanced node with more initial energy. The advanced node is more likely to become a $\mathrm{CH}$ than a normal node. In general, as the initial energy of the network increases, the overall lifetime of the network also increases, and the instability period decreases. However, factors such as energy and distance are not taken into account in the $\mathrm{CH}$ election process so that the energy consumption of $\mathrm{CH}$ is not balanced.

In the improved scheme of intercluster communication, $\mathrm{Hu}$ and Xiao constructed intercluster multihop routing by combining the idea of node chain in the PEGASIS protocol [22]. Finally, the node acting as a Leader on the chain undertakes the task of communication with the BS. The protocol reduces the energy consumed by direct communication between multiple $\mathrm{CHs}$ and the $\mathrm{BS}$ in the $\mathrm{LEACH}$ protocol. At the same time, the multihop transmission between $\mathrm{CH}$ s also enhances the scalability of the network. Li et al. considered the "hot spot" problem in multihop wireless sensor networks [23]. The hot spot problem refers to that the $\mathrm{CH}$ closer to the BS needs to bear more relay traffic than other $\mathrm{CHs}$, leading to the premature death of the $\mathrm{CH}$ closer to the BS, resulting in the exposure of the network area and partitioning of network. Thus, the author divides the nodes into clusters of different sizes. The cluster radius increases with the distance between the $\mathrm{CH}$ and the BS. In this way, the $\mathrm{CHs}$ close to the BS bear less relay traffic which relatively balances the network energy consumption and extends the network life. Furthermore, in [24], the MHT-LEACH method proposed by Emad Alnawafa can determine whether it is possible to transmit their data to BS through intermediate nodes according to its location and distance from BS. Compared with the LEACH protocol, MHT-LEACH improves network life, stability, and throughput. Then, Alnawafa and Marghescu proposed the IMHT-LEACH method in [25] to solve the problem that MHT-LEACH requires a large amount of energy to transfer data to the $\mathrm{CH}$ of the inner group. IMHT-LEACH can reduce the energy cost of data transfer to BS, especially in the huge deployment area or the large distance from the sensor area to BS. Compared with LEACH and MHT-LEACH protocols, IMHT-LEACH protocol can prolong the lifetime, improve the stability period, and increase the throughput of the sensor network.

These protocols significantly reduce energy consumption in the data transmission phase but consume much energy in the process of establishing routing. While ensuring high efficiency, the efforts must be taken to avoid consuming too much energy in establishing routes. Therefore, we have made the following contributions:

(1) First of all, we consider the factors such as energy in the cluster head election stage and the clustering stage, solve the energy balance and "hot spot" problems, and reduce the risk of premature death of the network.

(2) Then, the regional autonomous intercluster multihop routing mechanism is adopted in the data transmission phase to solve the problem of premature death of nodes and achieve the purpose of balancing network energy consumption.

(3) Finally, simulation experiments are carried out on the protocol, and the results show that the network lifetime, energy consumption rate, stability, and throughput have been improved.

\section{FOI-LEACH Routing Protocol}

Compared with WSNs, the network of field observation instruments is connected with data acquisition equipment by network nodes. Data acquisition instruments are generally connected with several or dozens of sensor devices [26], which can observe a large number of observation elements. It has the characteristics of large data storage, complex network structure, and so on. The network has relatively few nodes which have strong processing capacity to process data and instructions, to complete the communication with the data acquisition equipment and other tasks. There is auxiliary power supply from solar cells for networking nodes, but because the field observation instrument networks are usually deployed in a harsh environment, the problem of energy consumption must be fully considered. Therefore, we proposed an improved routing protocol based on LEACH protocol to meet the needs of the field observation instrument networking.

3.1. Improvement in $\mathrm{CH}$ Election Threshold. At the stage of $\mathrm{CH}$ election, the random election method of the original LEACH protocol may let the nodes with less energy be selected as $\mathrm{CHs}$, leading to the premature death of networking nodes. To solve the energy consumption problem in the harsh environment, the energy factor of the node was considered, and the concept of "self-energy" was introduced to recharge the node. With the continuous development and progress of environmental energy collection technology, researchers have been able to collect energy in the environment, such as solar light, mechanical vibration, and heat, and apply them to WSN [27] design sensors that can automatically collect environmental energy. The sensor node can convert the solar energy, vibration energy, and thermal energy in the environment into usable electric energy and automatically recharge energy for itself, forming a WSN with the characteristics of self-energy [28]. In order to achieve long-term observation and data transmission, we adopted the solar cell power supply in the field observation instrument network so that the energy of network nodes can be 
recharged. Correspondingly, the election threshold of $\mathrm{CHs}$ is modified to reduce the possibility that nodes with lower energy are elected as $\mathrm{CHs}$.

The threshold formula of node $i$ in round $r$ is shown as follows:

$$
T(i)= \begin{cases}\frac{p_{o p t}}{1-p_{\mathrm{opt}} \times \bmod \left(r, \operatorname{round}\left(1 / p_{\mathrm{opt}}\right)\right)} & \\ \times \frac{E_{r}(i)+E_{h}(i, r-1)}{E_{o}}, & i \in G, \\ 0, & i \notin G,\end{cases}
$$

where $p_{\text {opt }}$ is the optimal probability of a node becoming a $\mathrm{CH}, E_{o}$ is the initial energy of the node, $E_{r}(i)$ is the residual energy of the current node, and $E_{h}(i, r-1)$ is the energy obtained by the current node in the previous round. Since the energy acquisition environment of each node is not constant, the energy acquisition rate of node $i$ in $k$ units of time is denoted as $\alpha_{i}(k)$, and the energy acquired by node $i$ in the $r-1$ round [29] is denoted in the following equation:

$$
E_{h}(i, r-1)=\sum_{k=T(r-1)}^{T(r)} \alpha_{i}(k) .
$$

According to the above analysis, in the $\mathrm{CH}$ election, the improved threshold should consider the total number of $\mathrm{CHs}$ needed by the network and the number of times each node has become a $\mathrm{CH}$, as well as the initial energy value of the node, the residual energy value of the node in the previous round, and the supplied energy value of the node. In the process of cluster establishment of the $r$ round, each node firstly needs to count the initial energy value and the current residual energy value and calculate the energy value obtained in the previous round according to the energy recharging status. If the residual energy and recharge energy of the node are both low, the probability of the node becoming a $\mathrm{CH}$ in the $r+1$ round is reduced to avoid its death due to excessive energy consumption. On the contrary, when the residual energy and replenishment energy of the node are sufficient, the probability of the node becoming a $\mathrm{CH}$ is increased.

3.2. Improvement in Clustering Method. After the election of the $\mathrm{CH}$, the clustering operation is performed. Since the LEACH protocol divides the network into clusters of equal size, the CHs near the BS not only have to bear the energy consumed by the data transmission within their clusters but also the forwarding energy brought by the $\mathrm{CHs}$ far away from the BS, which leads to unbalanced traffic load between nodes and form a "hot spot" problem. In order to alleviate and avoid the "hot spot" problem, an energy-efficient and balanced network clustering routing protocol for observation instruments in harsh areas is constructed. In the routing protocol, clusters of unequal sizes are constructed, and the size of the cluster is proportional to the distance between the $\mathrm{CH}$ and the BS. In other words, the closer it is to the BS, the smaller the cluster is, and it consumes less energy in the communication within the cluster and more energy in the intercluster communication. On the contrary, the farther away from the BS, the larger the cluster is, and the more the energy it consumes is concentrated in the communication within the cluster. Moreover, since the node has different energy levels in the heterogeneous network, not only the distance between the node and the BS but also the current residual energy and recharge energy of the node should be considered to calculate the competitive radius [30], which was shown in the following equation:

$$
R_{c}=\left[1-\alpha \times \frac{d_{\max }-d(i, B S)}{d_{\max }-d_{\min }}-\beta \times\left(1-\frac{E_{r}(i)+E_{h}(i, r-1)}{E_{o}}\right)\right] \times R_{c}^{0},
$$

in which $\alpha$ and $\beta$ are the weighted factors of the value in [0, $1]$ and satisfy the condition that $\alpha+\beta=1 . R_{c}^{0}$ is the maximum competitive radius. $d_{\max }$ and $d_{\min }$ are the maximum and minimum values from nodes to BS in the network. $d(i, B S)$ is the distance between the current node $i$ and the BS. By dividing the network into clusters of different sizes according to the distance from the nodes to the BS, the current residual energy, and the supply energy of the nodes, the $\mathrm{CHs}$ near or far away the BS consume approximately equal energy, so as to achieve the purpose of energy balance in the field instrument network.

\subsection{Improvement in Communication Method. After the} election of $\mathrm{CHs}$ and the formation of clusters, the network enters the stable transmission stage. In general, the time of stable transmission is longer than that of cluster formation. In the LEACH protocol, data transmission is a single-hop transmission. That is, all member nodes send the collected data information to the $\mathrm{CH}$ of the cluster, and the $\mathrm{CH}$ sends it to the BS after data fusion. This causes the $\mathrm{CH}$ far away from the BS to consume more energy so that it may die in the transmission process and fail to transmit data successfully, which significantly reduces the network efficiency. Therefore, a multihop approach to data transmission was considered, and the concept of "zone" was introduced in this paper. The zone especially refers to the ring bands formed by taking the BS as the center of the circle and the adjacent circumferential lines with different lengths as the radius. A zone can contain several clusters, and a cluster must belong to a specific zone [8]. The BS controls the transmission energy, broadcasts the information of different intensities, and divides the monitoring area into three-ring zones $A, B$, and $C$, as shown in Figure 2. The data communication method adopts the principle of nearest partitioning; that is, the $\mathrm{CH}$ in zone $\mathrm{C}$ selects the $\mathrm{CH}$ closest to it in zone $B$ as the next hop. Similarly, the $\mathrm{CH}$ in zone $B$ selects the nearest $\mathrm{CH}$ in zone $A$ as the next hop. The $\mathrm{CH}$ in zone $A$ communicates directly with the BS. According to the characteristics of partitioning, because cluster nodes in zone $A$ and zone $B$ need to bear more energy consumed by data fusion and forwarding, only the nodes in zone $A$ and zone $B$ are powered by solar cells. 


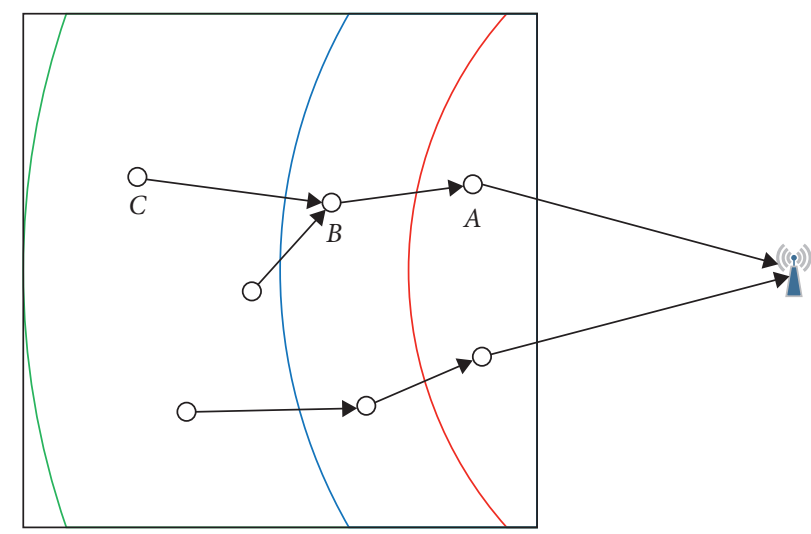

FIgURe 2: Partitioning method in the routing protocol.

3.4. Analysis of Heterogeneity. In this paper, in order to prolong the stable stage of the network, we adopted the value of heterogeneity set in literature [21] to set up two kinds of nodes with different initial energies, namely, the normal nodes with lower initial energy and the advanced nodes with higher initial energy. Advanced nodes are more likely to become CHs and not easy to die than normal nodes, which is to balance energy consumption. At the same time, the total energy of the network has changed. If the initial energy of the normal node is $E_{o}$, the initial energy of the advanced node is $(1+a) \cdot E_{o}$. So the total energy of the network is changed to $(1-m) \cdot E_{o}+m \cdot(1+a) \cdot E_{o}=(1+a \cdot m) \cdot E_{o}$; that is, the total energy of the network has increased by $1+$ a.m times. Accordingly, in the $\mathrm{CH}$ election, the optimal probability of the node becoming the $\mathrm{CH}$ has also been changed. The weighted probabilities of normal nodes and advanced nodes are shown in the following equations, respectively:

$$
\begin{aligned}
p_{\text {nrm }} & =\frac{p_{\mathrm{opt}}}{1+\alpha \cdot m} \\
p_{\mathrm{adv}} & =\frac{p_{\mathrm{opt}}}{1+\alpha \cdot m} \times(1+a) .
\end{aligned}
$$

The optimal $\mathrm{CH}$ number is $k_{\mathrm{opt}}=\sqrt{n / 2 \pi} \cdot \sqrt{E_{f s} / E_{m p}}$. $m / d_{\text {to }}^{2} B S$ [30], where $k_{\text {opt }}$ represents the optimal cluster number, $n$ is the number of nodes set in the monitoring area $M \times M$, and $d_{t o} B S$ represents the distance between the $\mathrm{CH}$ and the BS.

For normal nodes, the modified election threshold is shown in equation (7). Similarly, the election threshold for advanced nodes is shown in equation (8):

$$
\begin{gathered}
T(i)= \begin{cases}\frac{p_{n r m}}{1-p_{n r m} \times \bmod \left(r, \operatorname{round}\left(1 / p_{n r m}\right)\right)} \times \frac{E_{r}(i)+E_{h}(i, r-1)}{E_{o}}, & i \in G, \\
0, & i \notin G,\end{cases} \\
T(i)= \begin{cases}\frac{p_{\mathrm{adv}}}{1-p_{\mathrm{adv}} \times \bmod \left(r, \operatorname{round}\left(1 / p_{\mathrm{adv}}\right)\right)} \times \frac{E_{r}(i)+E_{h}(i, r-1)}{E_{o}}, & i \in G, \\
0, & i \notin G .\end{cases}
\end{gathered}
$$

In the above two threshold equations, it can be found that the weighted probabilities are different because the advanced node has more initial energy. Due to the energy factor is taken into account when electing the $\mathrm{CH}$, the higher the energy is, the easier it is to become the $\mathrm{CH}$, so the advanced nodes are more likely to become the $\mathrm{CH}$ s than the normal nodes.

3.5. FOI-LEACH Routing Protocol. FOI-LEACH protocol introduces the concepts of "self-energized" and "zone" combined with the characteristics of field instrument networking. In addition, the $\mathrm{CH}$ election stage and cluster formation stage are carried out in each zone, respectively. The flow of each round of the FOI-LEACH protocol is shown in Figure 3, and the specific operation steps are described as follows:

(1) After the deployment of the network nodes, each node estimates the distance from the BS according to the signal strength transmitted by the BS, judges, and identifies the zone to which it belongs.

(2) At the beginning of the $\mathrm{CH}$ election, the node generates a random number from 0 to 1 , counts the current residual energy $E_{r}(i)$ and the previous round of energy replenishment $E_{h}(i, r-1)$, and determines 


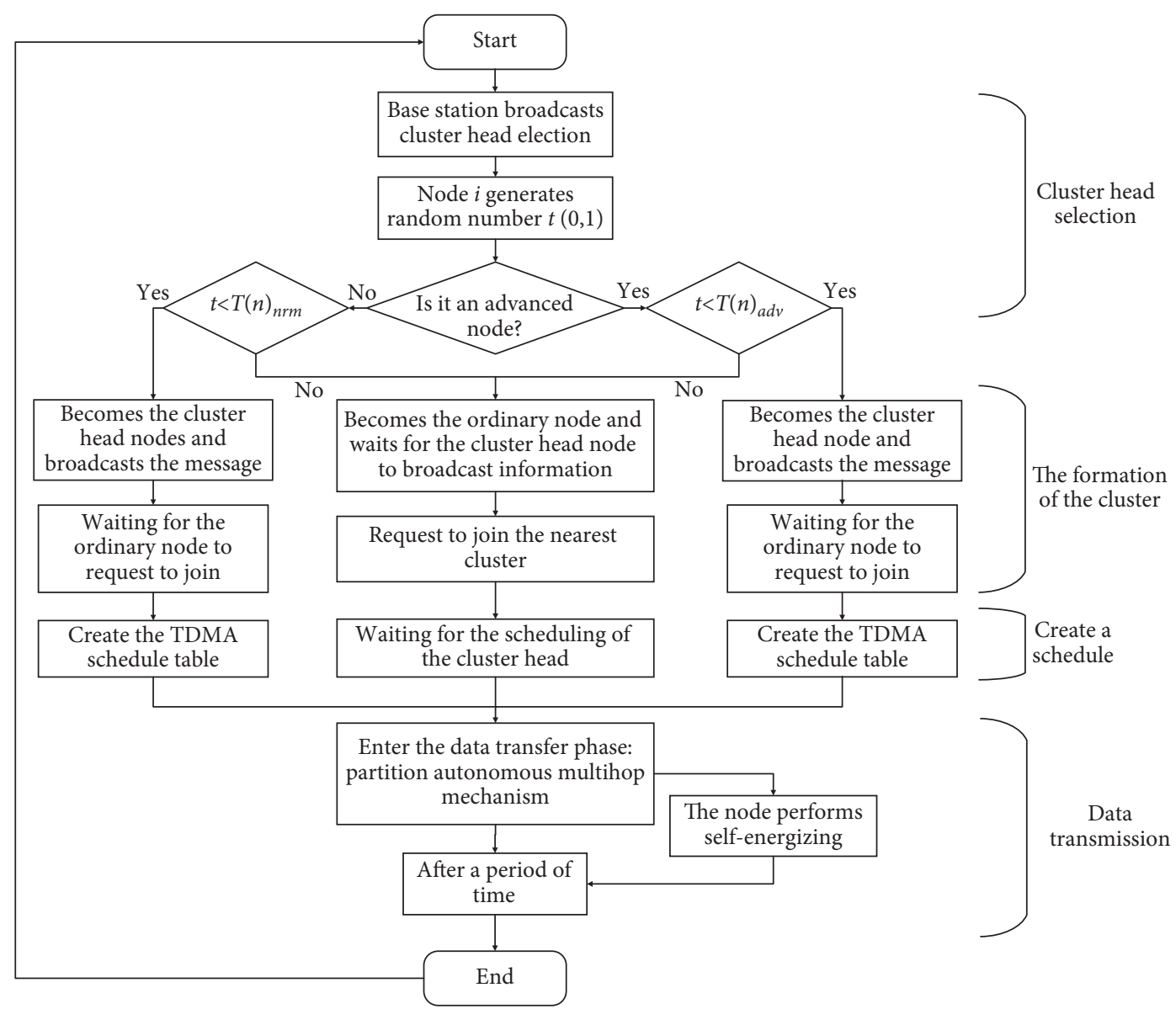

FIGURE 3: Flow chart of operation in each round of FOI-LEACH protocol.

whether the node is an advanced node. Moreover, if it is an advanced node, it calculates the threshold, as shown in equation (8); otherwise, it calculates the threshold, as shown in equation (7), and then judges whether the random number generated by the node is lower than the threshold; if so, announce the message that it becomes the $\mathrm{CH}$; otherwise, it becomes a member node and waits for the scheduling of the $\mathrm{CH}$.

(3) After the $\mathrm{CH}$ election, according to the $\mathrm{BS}$ radio message, all the nodes maximum distance $d_{\max }$ and minimum $d_{\min }$ of network nodes to the BS and the distance $d(i, B S)$ of the current node to the BS are statistically counted, and the current residual energy of the node $E_{r}(i)$ and the energy supply of the previous round $E_{h}(i, r-1)$ are calculated, and then, cluster competition radii are computed. The node detects the received announcement message and determines whether the $\mathrm{CH}$ exists within the competition radius of the zone. If so, a $\mathrm{CH}$ is selected according to the competition radius to join and form a cluster. If there is no $\mathrm{CH}$ within the competitive radius, it automatically becomes a $\mathrm{CH}$ and publishes a notice.

(4) After the cluster is formed, the $\mathrm{CH}$ creates a TDMA schedule, and the member nodes in the cluster wait for the $\mathrm{CH}$ to be scheduled.
(5) In the data transmission stage, cluster member nodes collect data regularly and send the data directly to the $\mathrm{CH}$ through the single-hop transmission. The $\mathrm{CH}$ compresses and fuses the received data and then sends it to the nearest $\mathrm{CH}$ in the next zone until it is transmitted to the BS. Loop through steps 1 to 5 until the network fails.

\section{Network Model of Field Observation Instruments}

4.1. Network Model. In this paper, we consider the random deployment of $n$ nodes in a square monitoring area with a size of $M \times M$. The assumptions for the networking environment are as follows:

All networking nodes have unique IDs and are heterogeneous.

All networking nodes are randomly deployed in the monitoring area and cannot be moved after deployment.

All networking nodes know their residual energy, and some nodes can supplement the energy.

All networking nodes can determine the distance from the information source according to the intensity of the received information. 
The BS is located outside the monitoring area, it has sufficient energy resources, and each node knows the location of the BS.

4.2. Energy Model. Energy consumption is an important criterion to evaluate the performance of a routing protocol. The network life mainly depends on the energy consumed in data sending and data receiving. Meanwhile, the energy consumed by the $\mathrm{CH}$ when using the fusion technology should be considered, and the energy consumption of the node in the process of calculation and storage should be neglected. LEACH protocol uses first-order wireless mode in WSNs, so it adopts a simple energy consumption model [12]. The model defines that the energy consumed by each $l$ bit data sent by the wireless circuit is

$$
\begin{aligned}
E_{\mathrm{Tx}}(l, d) & =E_{\mathrm{Tx}-\mathrm{elec}}(l, d)+E_{\mathrm{Tx}-\mathrm{amp}}(l, d) \\
& = \begin{cases}l E_{\mathrm{elec}}+l E_{\mathrm{fs}} d^{2}, & d<d_{0}, \\
l E_{\mathrm{elec}}+l E_{\mathrm{mp}} d^{4}, & d \geq d_{0} .\end{cases}
\end{aligned}
$$

When receiving information, the energy consumed by the wireless circuit receiving $l$ bit data is

$$
E_{\mathrm{Rx}}(l, d)=E_{\mathrm{Rx}-\mathrm{elec}}(l, d)=l E_{\mathrm{elec}} .
$$

The energy consumed by data fusion is

$$
E_{\mathrm{Gx}}=l E_{\text {gather }} \text {. }
$$

In equations (9)-(10), $d$ represents the distance between the sending node and the receiving node and $E_{\text {elec }}$ represents the energy consumed for each bit of data sent or received. In equation (9), $E_{\mathrm{fs}} d^{2}$ is the energy consumed by the free space model for each $l$ bit of data amplification, and $E_{\mathrm{mp}} d^{4}$ is the energy consumed by the multipath attenuation model for each $l$ bit of data amplification. Obviously, when the distance between nodes becomes more extensive, the energy consumption increases exponentially. Therefore, if you want to reduce the energy consumption of data transmission, you must reduce the distance between nodes. The $E_{\text {gather }}$ in equation (11) is expressed as the energy consumed by data fusion for each processing of $1 \mathrm{bit}$; the $d_{0}$ in equation (9) is a threshold, which is determined by the equation (12), and is defined as $87 \mathrm{~m}$ in this paper:

$$
d_{0}=\sqrt{\frac{E_{f s}}{E_{m p}}} .
$$

\section{Simulation Experiments and Analysis}

5.1. Experimental Setting. In order to analyze the performance of the FOI-LEACH protocol, this paper conducts simulation experiments on MATLAB simulation platform to compare it with the original LEACH protocol [4], SEP protocol [21], and PECRP protocol [31]. The simulation experiments were carried out under the monitoring area of $100 \mathrm{~m} \times 100 \mathrm{~m}$, and the BS is located outside the monitoring area with the coordinate of $(150,50)$. As shown in Figure 4,

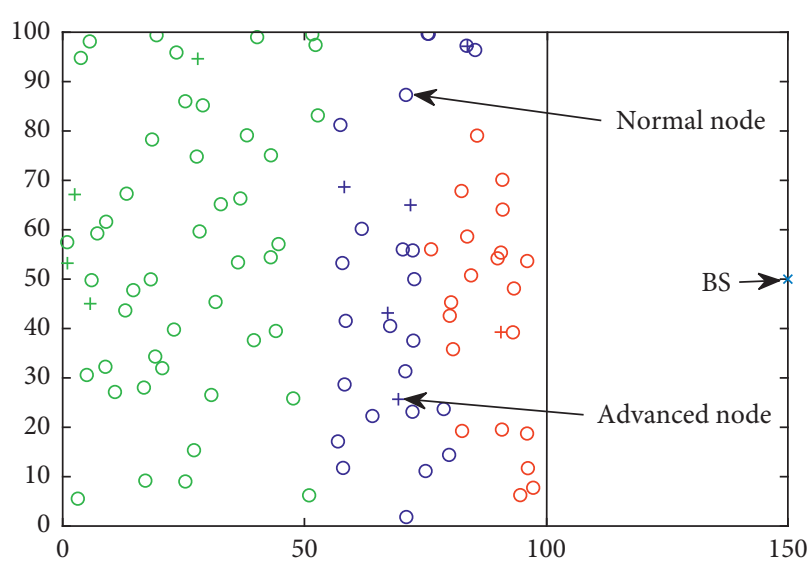

FIgURE 4: Distribution diagram of initial nodes.

the initial node distribution map distinguishes the nodes in each zone by color, in which red represents the nodes in zone $A$, blue represents the nodes in zone $B$, and the green

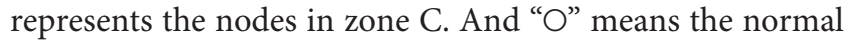
node, "+" means the advanced node, and " $x$ " means BS.

In the self-energy experiment, all the surviving nodes in the network are replenished with energy once every five rounds until the simulation end. The recharge energy is distributed randomly to all remaining surviving nodes in the network. The total energy of the first replenishment is set to $0.35 \mathrm{~J}$. In the later replenishment, in order to prevent the nodes in the network from dying when there are too few nodes. We set the attenuation factor alpha to 0.9; that is, the energy replenishment to all the nodes in the network is 0.9 times of the previous time. The initial energy and topology of all the experimental networks were set to the same environment. When all the nodes in the network die, the network is considered to be invalid. Experiments proved that the network works best when the parameters are set to the following values: $m$ is set to $0.1, a$ is set to $2, \alpha$ is set to 0.3 , and $\beta$ is set to 0.7 . The range of optimal $\mathrm{CH}$ number is $[1,14]$, and 10 is taken in this paper; the probability of optimal $\mathrm{CH}$ number is $p_{\mathrm{opt}}=0.1$. The three-partition system shown in Figure 2 was adopted in the routing protocol. The specific parameters of the simulation experiments are listed in Table 1.

5.2. Determination of the Partitioning Scheme. The FOI$\mathrm{LEACH}$ protocol proposed in this paper is based on the improvement of the LEACH protocol. In the initial stage of node deployment, the distance between the node and the BS is obtained according to the signal strength of the BS received by the node. Then, the monitoring area is partitioned according to the distance. To determine the optimal partitioning scheme in the FOI-LEACH protocol, we proposed the following five schemes for the protocol and tested them in the partitioning experiment:

Scheme 1. The monitoring area is divided into two zones $A$ and $B$ and the zone $S_{A}$ of $A$ is equal to the zone $S_{B}$ of $B$, as shown in Figure 5 (partitioning scheme 1). The $\mathrm{CH}$ in zone $\mathrm{B}$ chooses the $\mathrm{CH}$ in zone $A$ closest 
TABle 1: Parameters in the simulation experiments.

\begin{tabular}{lc}
\hline Parameter & Value \\
\hline Total number of nodes $N$ & 100 \\
Network area (m) & $100 \times 100$ \\
Coordinates of BS (m) & $(150,50)$ \\
Data packet size $L$ (bit) & 4000 \\
Initial energy of normal node $(\mathrm{J})$ & 0.1 \\
Initial energy of advanced node $(\mathrm{J})$ & 0.3 \\
Energy consumption of transmitting circuit $E_{T X}(\mathrm{~nJ} /$ & 50 \\
bit) & \\
Energy consumption of receiving circuit $E_{R X}(\mathrm{~nJ} / \mathrm{bit})$ & 50 \\
Free space model energy $E_{f s}\left(\mathrm{pJ} / \mathrm{bit} / \mathrm{m}^{2}\right)$ & 10 \\
Multipath attenuation model energy $E_{m p}\left(\mathrm{pJ} / \mathrm{bit} / \mathrm{m}^{2}\right)$ & 0.0013 \\
Energy consumption of data fusion $E_{D A}(\mathrm{~nJ} / \mathrm{bit})$ & 5 \\
Probability of the optimal number of CHs $p_{\mathrm{opt}}$ & 0.1 \\
Maximum cluster radius in zone $A R_{c A}(\mathrm{~m})$ & 12.5 \\
Maximum cluster radius in zone $B R_{c B}(\mathrm{~m})$ & 12.5 \\
Maximum cluster radius in zone $C R_{c C}(\mathrm{~m})$ & 25 \\
Attenuation factor alpha & 0.9 \\
$M$ & 0.1 \\
$A$ & 2 \\
$\alpha$ & 0.3 \\
$\beta$ & 0.7 \\
\hline
\end{tabular}

to it for forwarding data, and the $\mathrm{CHs}$ in zone $A$ communicates directly with the BS.

Scheme 2. As shown in Figure 5 (partitioning scheme 2), the monitoring area is divided into two zones $A$ and $B$ and the $S_{A}$ of zone $A$ is smaller than the area $S_{B}$ of zone $B$.

Scheme 3. The monitoring area is divided into three zones $A, B$, and $C$, and the area $S_{A}$ of zone $A$ is equal to the area $S_{B}$ of zone $B$ and smaller than the area $S_{C}$ of zone $C$, as shown in Figure 5 (partitioning scheme 3).

Scheme 4. The monitoring area is divided into three zones $A, B$, and $C$, and the area $S_{A}$ of zone $A$ is smaller than the area $S_{B}$ of zone $B$ and smaller than the area $S_{C}$ of zone $C$, as shown in Figure 5 (partitioning scheme 4).

Scheme 5. The monitoring area is divided into three zones $A, B$, and $C$, and the area $S_{A}$ of zone $A$ is equal to the area $S_{C}$ of zone $C$ and smaller than the area $S_{B}$ of zone $B$, as shown in Figure 5 (partitioning scheme 5).

The survival nodes and the average residual energy in each round of the five partitioning schemes are drawn in Figures 6 and 7, respectively. Based on the experimental analysis, it can be found that Scheme 3 indicated as a red curve in the figures has a better performance. In scheme 3, i.e., $S_{A}=S_{B}<S_{C}$, the first dead node appears in the 587th round, which is later than other schemes; that is, the stability period is more extended than other schemes. The average residual energy consumption rate of nodes is also lower than that of other schemes. This is because zones $A$ and $B$ need to bear more forwarding energy, so when these two zones are smaller than zone $C$, the network energy consumption is more balanced. Therefore, we adopted the partitioning method of scheme 3 to carry out the simulation experiments.
5.3. Analysis of Parameters. In equation (4), parameters $\alpha$ and $\beta$ determine the difference in cluster size. Therefore, the relationship between these two parameters and network life was observed by taking $\alpha$ from 0 to 1 and $\beta$ from 1 to 0 . The results of parameters analysis are shown in Figure 8, which also proves the rationality of our unequal clustering mechanism. When parameter $\alpha$ increases from 0 to 1 , the effect of the unequal clustering method is noticeable. However, the impact of parameter $\beta$ on the network should also be considered. If the value of $\alpha$ is too large, the lifetime of the system will be shortened. It is because too many clusters will be generated near the BS, and each cluster sends packets to the BS which results in the waste of energy. Therefore, it needs to determine the optimal values for parameters $\alpha$ and $\beta$. In this experiment, we can conclude from Figure 8 that when $\alpha=0.3$ and $\beta=0.7$, the death time of the first node is later and the network is more stable.

5.4. Experimental Comparison Analysis. This paper mainly evaluates the performance of the observation instrument network routing protocol with and without energy supply through the following evaluation elements:

Network lifetime: the network lifetime is measured by the survival rate of the whole network nodes, which generally represents a certain proportion of the energy loss of the network node. In this paper, we took the death time interval from the beginning of the network to the last surviving node as the lifetime of the network. Energy consumption rate: it usually refers to the average energy consumption of a node sending or receiving a byte of data. It is essential to evaluate the performance of routing algorithms.

Stability: the stability of the field instruments observation network was evaluated from two aspects: the time interval from the beginning of the network to the death of the first node and the number of $\mathrm{CHs}$.

Network throughput: the indicator to measure the efficiency of the network, which is usually judged by the total number of packets sent to BS and the total number of packets sent to $\mathrm{CH}$.

\subsubsection{Experiments without Energy Supply}

(1) Network Lifetime. Figure 9 shows the number of surviving nodes in the network when the network runs for 2100 rounds without being self-energized. According to the statistics of the number of rounds of death node in Table 2, the network failure of the LEACH protocol occurs at round 276; the network of SEP protocol expires at 462 rounds and network failure of the PECRP protocol at round 438 . However, the FOI-LEACH protocol was not expired until round 2042, which significantly increased the network survival time. It is because the FOI-LEACH protocol considers such factors as energy and distance in stages of $\mathrm{CH}$ election, cluster formation, and intercluster communication. 


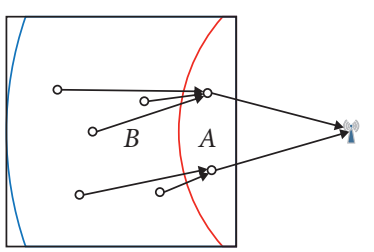

(a)

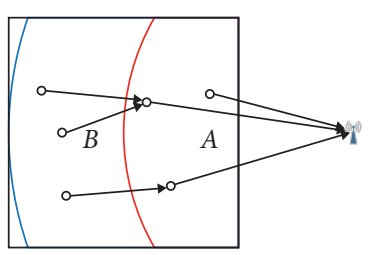

(b)

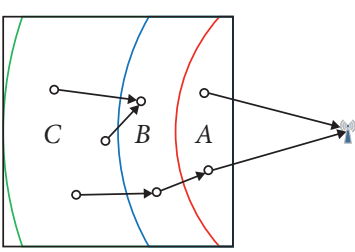

(c)

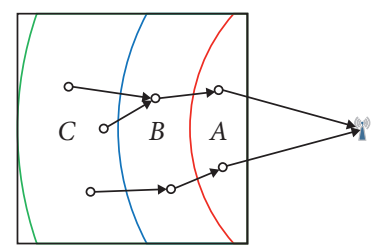

(d)

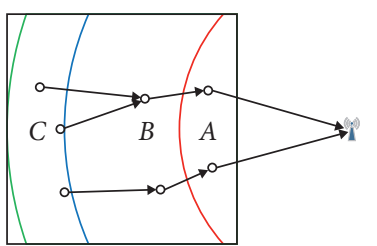

(e)

FIgure 5: Partitioning scheme. (a) Partitioning scheme 1: $S_{a}<S_{b}$. (b) Partitioning scheme 2: $S_{a}=S_{b}$. (c) Partitioning scheme 1: $S_{a}=S_{b}<S_{c}$. (d) Partitioning scheme 1: $S_{a}<S_{b}<S_{c}$. (e) Partitioning scheme 1: $S_{a}=S_{c}<S_{b}$.

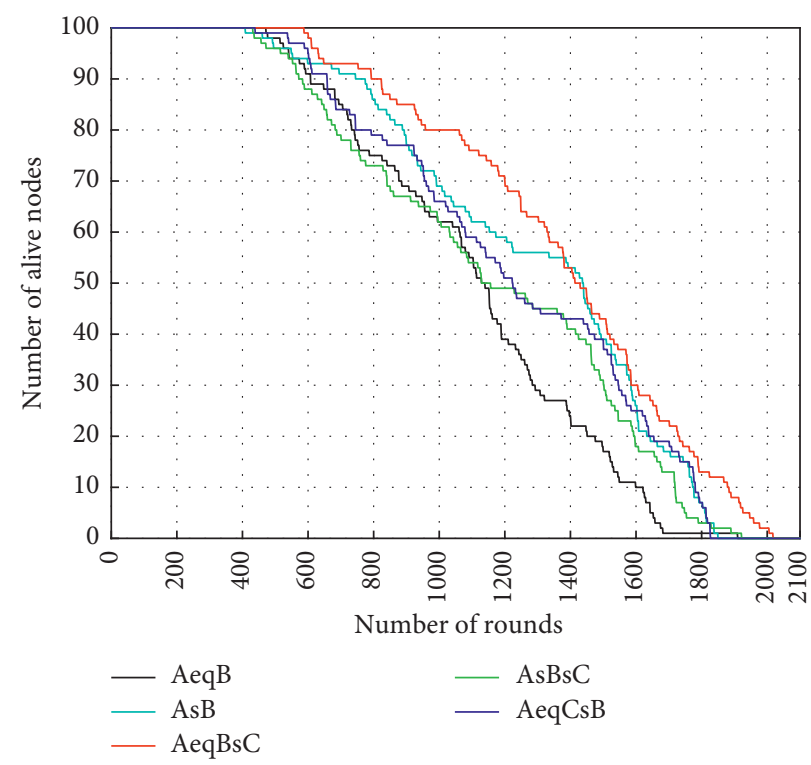

Figure 6: Comparison of active nodes in each round.

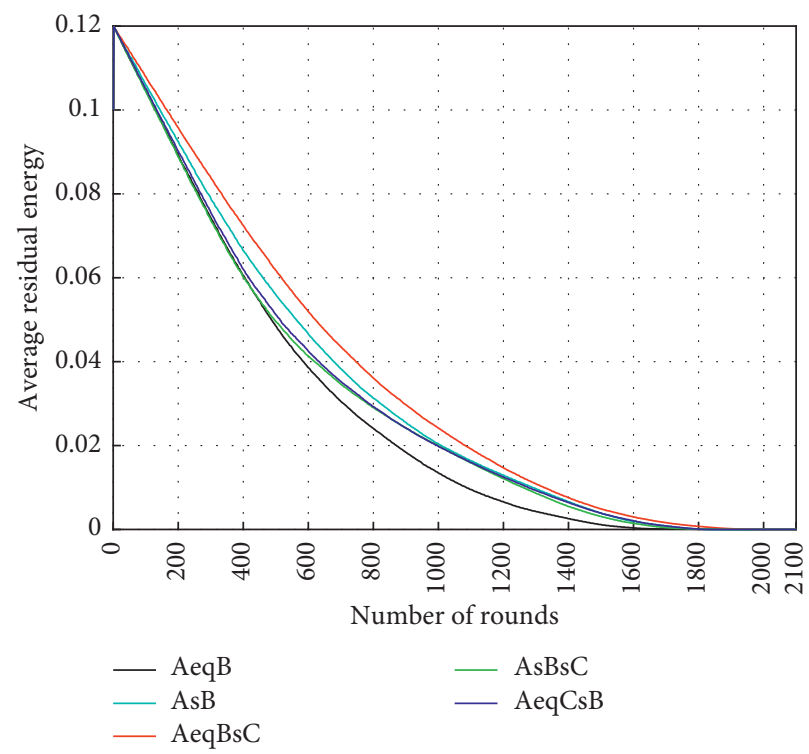

Figure 7: Comparison of average residual energy in each round. 


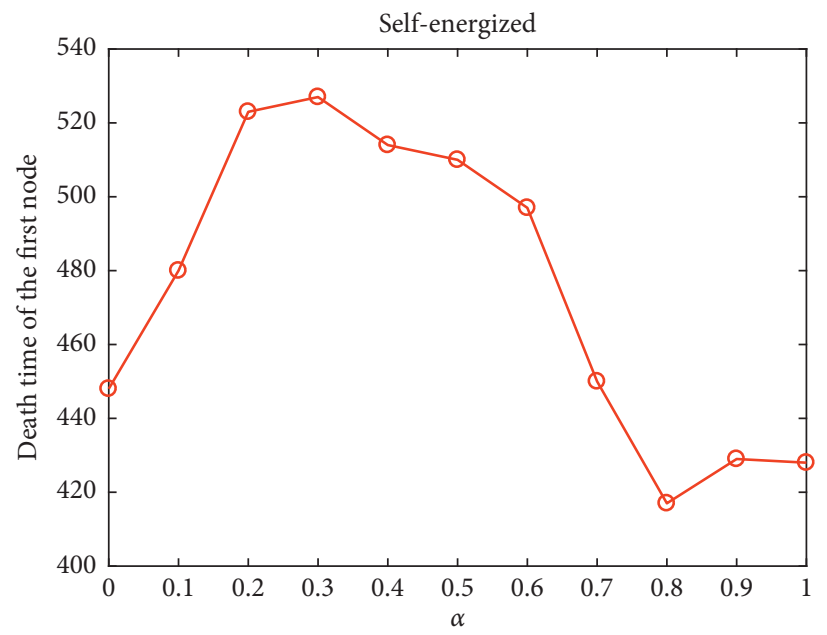

FIgURE 8: Impact of parameters $\alpha$ and $\beta$ on the network lifetime.

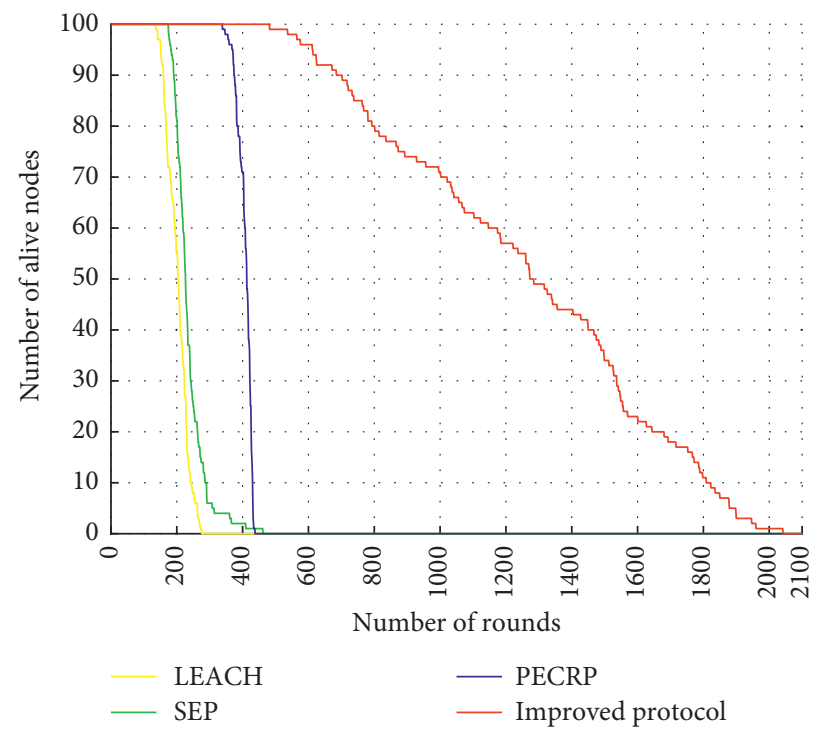

FIgURE 9: Comparison of live nodes in each round without power supply.

TABLE 2: Statistics number of rounds of dead nodes of the four protocols without power supply.

\begin{tabular}{lcccc}
\hline Protocol & First node dies & $10 \%$ of nodes die & $50 \%$ of nodes die & $100 \%$ of nodes die \\
\hline LEACH & 135 & 160 & 204 & 276 \\
SEP & 174 & 191 & 226 & 462 \\
PECRP & 338 & 374 & 412 & 438 \\
FOI-LEACH & 481 & 684 & 1272 & 2042 \\
\hline
\end{tabular}

(2) Average Residual Energy and Energy Dissipation Rate. Figure 10 shows the graph of the average residual energy of the network running 2100 rounds without being self-energized. Combined with the network average residual energy, which is shown in Table 3, it can be drawn that the FOILEACH protocol is more slowly energy consumed. Besides, it can be seen from Figure 10 that the energy consumption rate of the improved network is significantly reduced to achieve the purpose of delaying energy consumption and extending network life.

(3) Stability. The stability of networking was evaluated from two aspects. First, it can be seen from Figure 9 that the FOILEACH protocol significantly extends the death time of the 


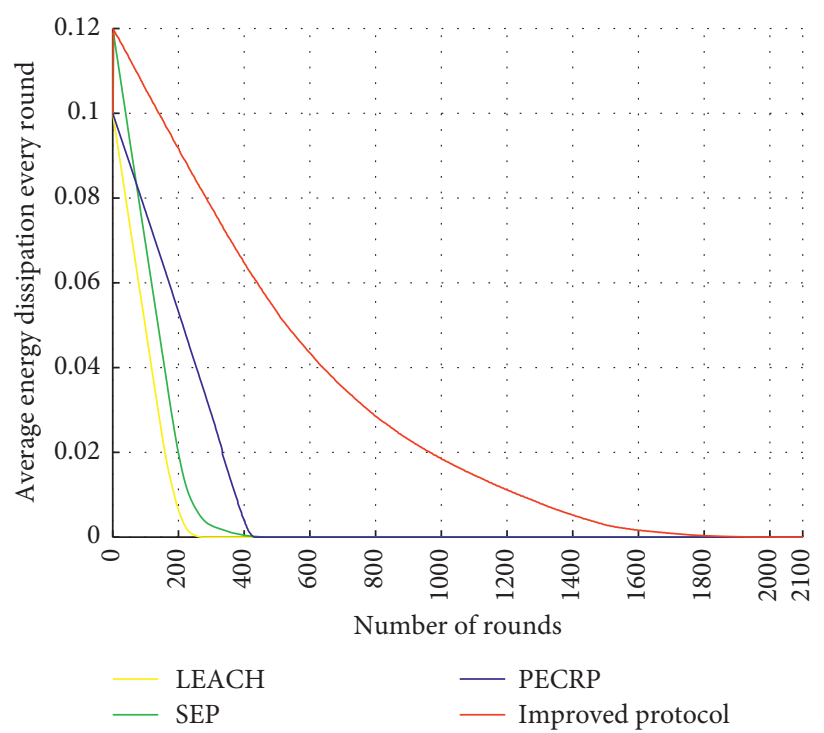

Figure 10: Comparison of average residual energy in each round without power supply.

TABLE 3: Statistics of the average residual energy (J) of nodes of the four protocols without power supply.

\begin{tabular}{lcccc}
\hline Protocol & 50 rounds & 200 rounds & 600 rounds & 2000 rounds \\
\hline LEACH & 0.075 & 0.007 & 0 & 0 \\
SEP & 0.094 & 0.02 & 0 & 0 \\
PECRP & 0.088 & 0.053 & 0 & 0 \\
FOI- & 0.113 & 0.092 & 0.043 & $1.52 \times 10^{-5}$ \\
LEACH & & & & \\
\hline
\end{tabular}

first node. For the LEACH protocol, the nodes began to die at round 135, SEP protocol at round 174, and PECRP protocol at round 338. Because the energy factor is taken into account in selecting the $\mathrm{CH}$, energy and distance factors are taken into account in clustering, and the adopted zonebased autonomous multihop communication model minimizes the Hello control package, the nodes die at round 481 for the FOI-LEACH protocol. As a result, the FOI-LEACH protocol has better performance than other protocols.

Figure 11 shows the distribution of the number of clusters of the four protocols without energy supply simulated for 10 rounds of random elections, in which Figure 11(a) represents the statistics number of $\mathrm{CHs}$ in each zone of the FOI-LEACH protocol and Figure 11(b) represents the statistics number of $\mathrm{CHs}$ in the whole network of FOI-LEACH protocol. Figures 11(c)-11(e) represent the statistics number of CHs of LEACH, SEP, and PECRP protocols, respectively. It can be seen that the number of CHs of FOI-LEACH protocol is more stable than that of LEACH, SEP, and PECRP. Because $\mathrm{LEACH}$ and SEP randomly select $\mathrm{CHs}$, the number of $\mathrm{CHs}$ produced was unstable. The FOI-LEACH protocol forms clusters by selecting $\mathrm{CHs}$ with a competitive radius, and the number of $\mathrm{CHs}$ is relatively stable.

(4) Network Throughput. Figure 12 shows that, in the LEACH protocol, SEP protocol, PECRP protocol, and our proposed FOI-LEACH protocol, the total number of packets sent to BS is approximately $900,2580,2980$, and 11140 , respectively. Moreover, Figure 13 shows that, in the LEACH protocol, SEP protocol, PECRP protocol, and FOI-LEACH protocol, the total number of packages sent to $\mathrm{CHs}$ is approximately $7500,21000,38200$, and 119000 , respectively. It is because the proposed scheme has a high network lifetime, the total number of packets sent to $\mathrm{BS}$ and $\mathrm{CHs}$ is much higher than other protocols.

\subsubsection{Experiments with Energy Supply}

(1) Network Lifetime. Figure 14 shows the number of surviving nodes in the network when the network runs for 2300 rounds self-energized. According to the statistics of death nodes in Table 4, we can find that the network lifetime of the four protocols all has been extended. The network failure of the LEACH protocol occurred at round 341; the network of the SEP protocol expires at round 665 and network failure of PECRP protocol at round 530; and the FOI-LEACH protocol was not expired until round 2246, which significantly increased the network survival time. It is demonstrated that the improved schemes can work well in both the environments with or without power supply.

(2) Average Residual Energy and Energy Dissipation Rate. Figure 15 shows the graph of the average residual energy of the network running 2300 rounds self-energized. Combined with the network average residual energy, which shown in Table 5, it can be drawn that the FOI-LEACH protocol is more slowly energy consumed. Besides, it can be seen from Figure 15 that the energy consumption rate of the improved network is significantly reduced to achieve the purpose of delaying energy consumption and extending network life.

(3) Stability. For the stability of field instruments networking, it can be seen from Figure 14 that the FOI-LEACH protocol significantly extends the death time of the first 

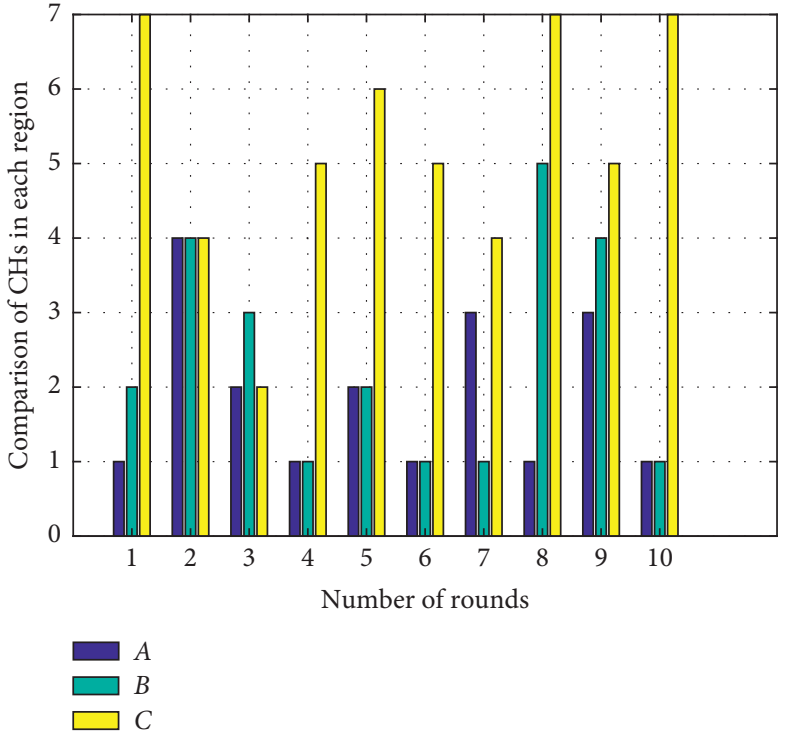

(a)

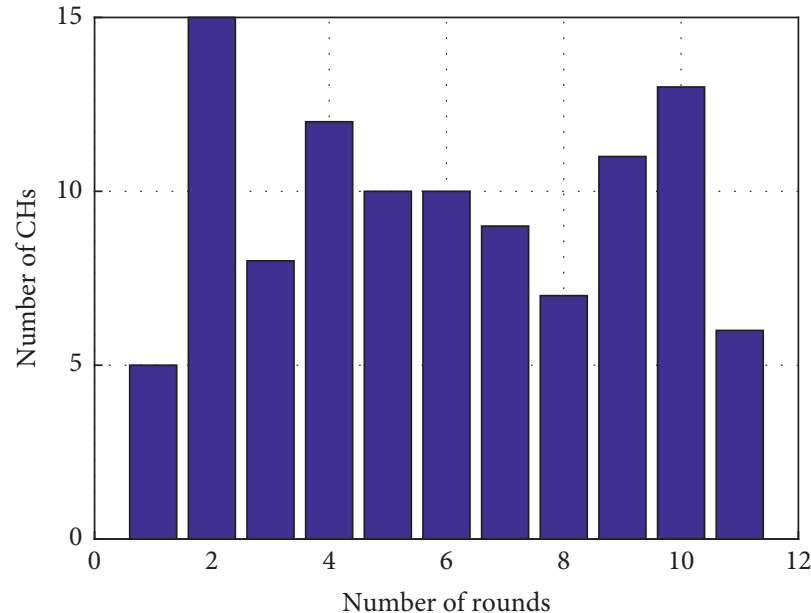

(c)

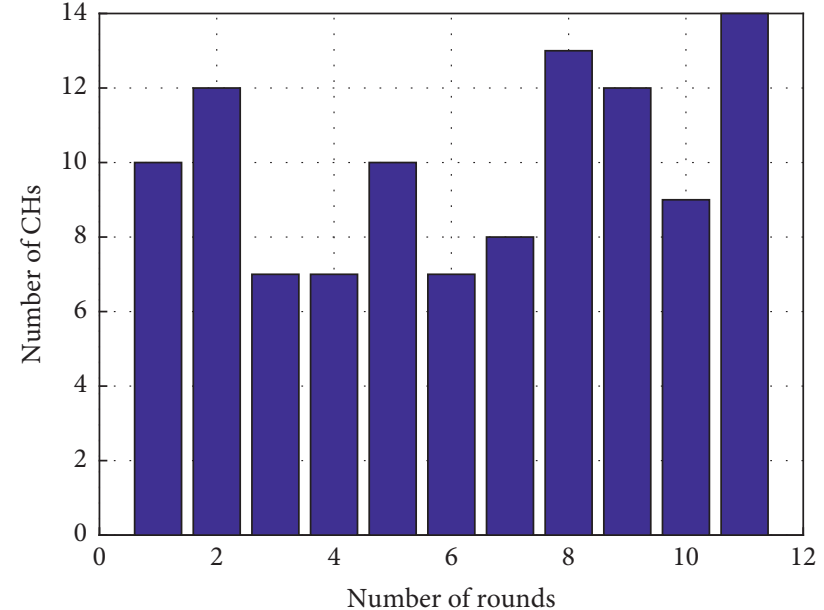

(b)

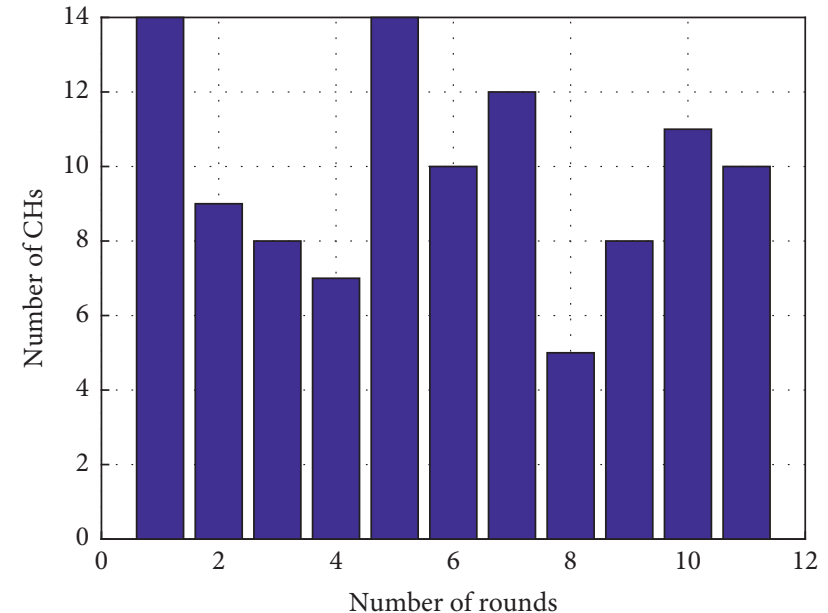

(d)

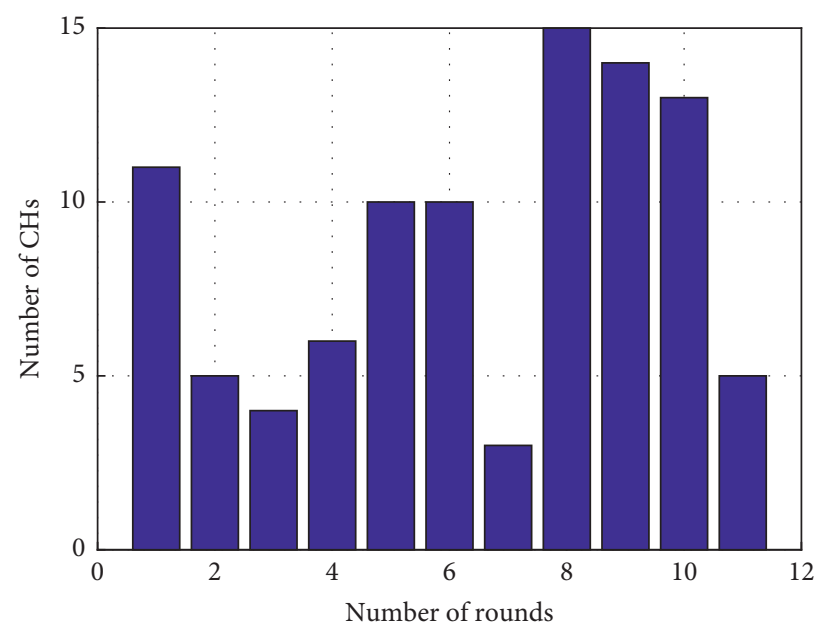

(e)

FIgUre 11: Statistics of CHs (a) in each zone of FOI-LEACH protocol, (b) of FOI-LEACH protocol, (c) of LEACH protocol, (d) of SEP protocol, and (e) of PECRP protocol without power supply. 


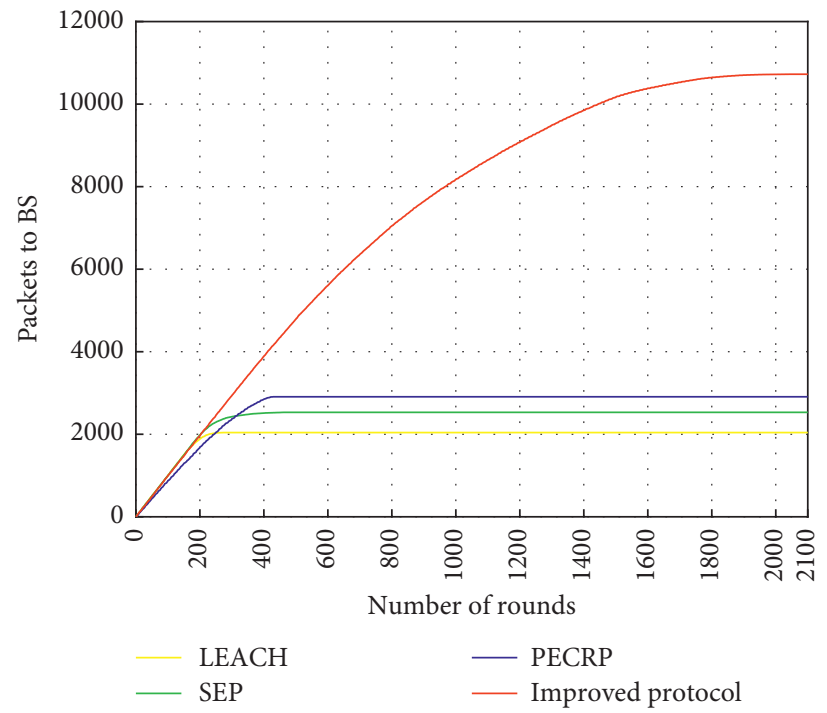

FIgURE 12: Comparison of packets sent to BS from CHs without power supply.

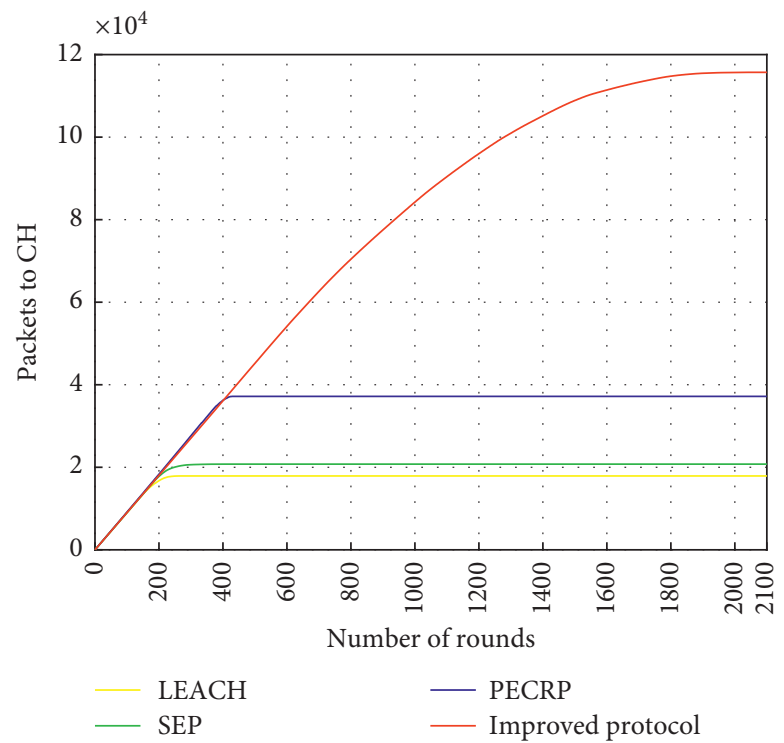

Figure 13: Comparison of packets sent to $\mathrm{CHs}$ from member nodes without power supply.

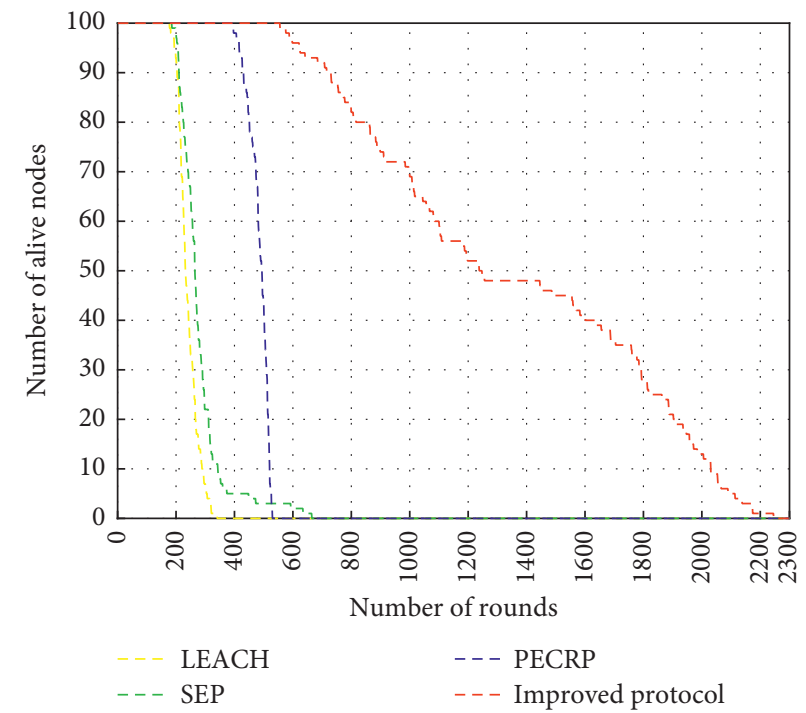

FIgURE 14: Comparison of live nodes in each round with power supply. 
TABLE 4: Statistics number of rounds of dead nodes of the four protocols with power supply.

\begin{tabular}{lcccc}
\hline Protocol & First node dies & $10 \%$ of nodes die & $50 \%$ of nodes die & $100 \%$ of nodes die \\
\hline LEACH & 177 & 204 & 232 & 341 \\
SEP & 185 & 210 & 264 & 665 \\
PECRP & 392 & 430 & 493 & 530 \\
FOI-LEACH & 555 & 716 & 1238 & 2246 \\
\hline
\end{tabular}

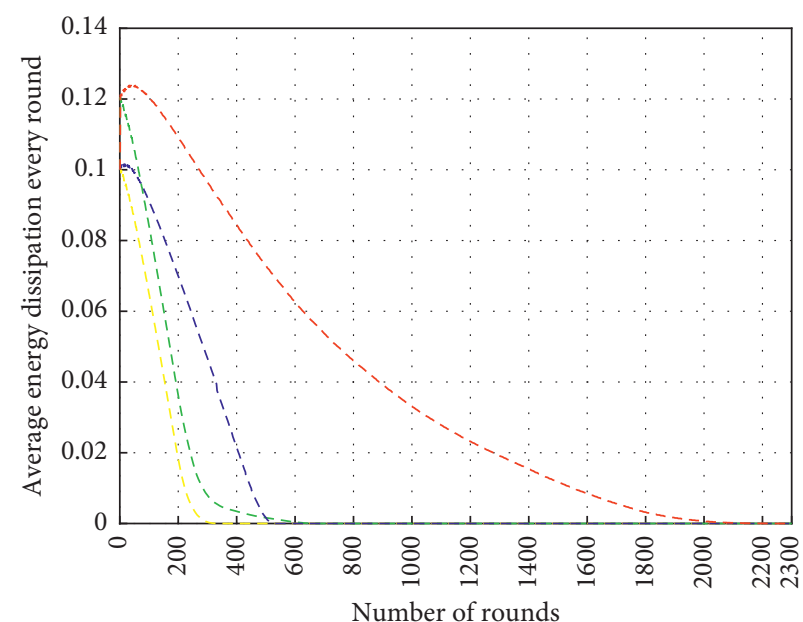

$$
\begin{array}{ll}
-- & \text { LEACH } \\
--- \text { SEP } & --- \text { PECRP } \\
& - \text { Improved protocol }
\end{array}
$$

FIGURE 15: Comparison of average residual energy in each round with power supply.

TABLE 5: Statistics of the average residual energy (J) of nodes of the four protocols with power supply.

\begin{tabular}{lcccc}
\hline Protocol & 50 rounds & 200 rounds & 600 rounds & 2000 rounds \\
\hline LEACH & 0.086 & 0.018 & 0 & 0 \\
SEP & 0.105 & 0.036 & 0.001 & 0 \\
PECRP & 0.099 & 0.07 & 0 & 0 \\
FOI-LEACH & 0.124 & 0.109 & 0.062 & 0.001 \\
\hline
\end{tabular}
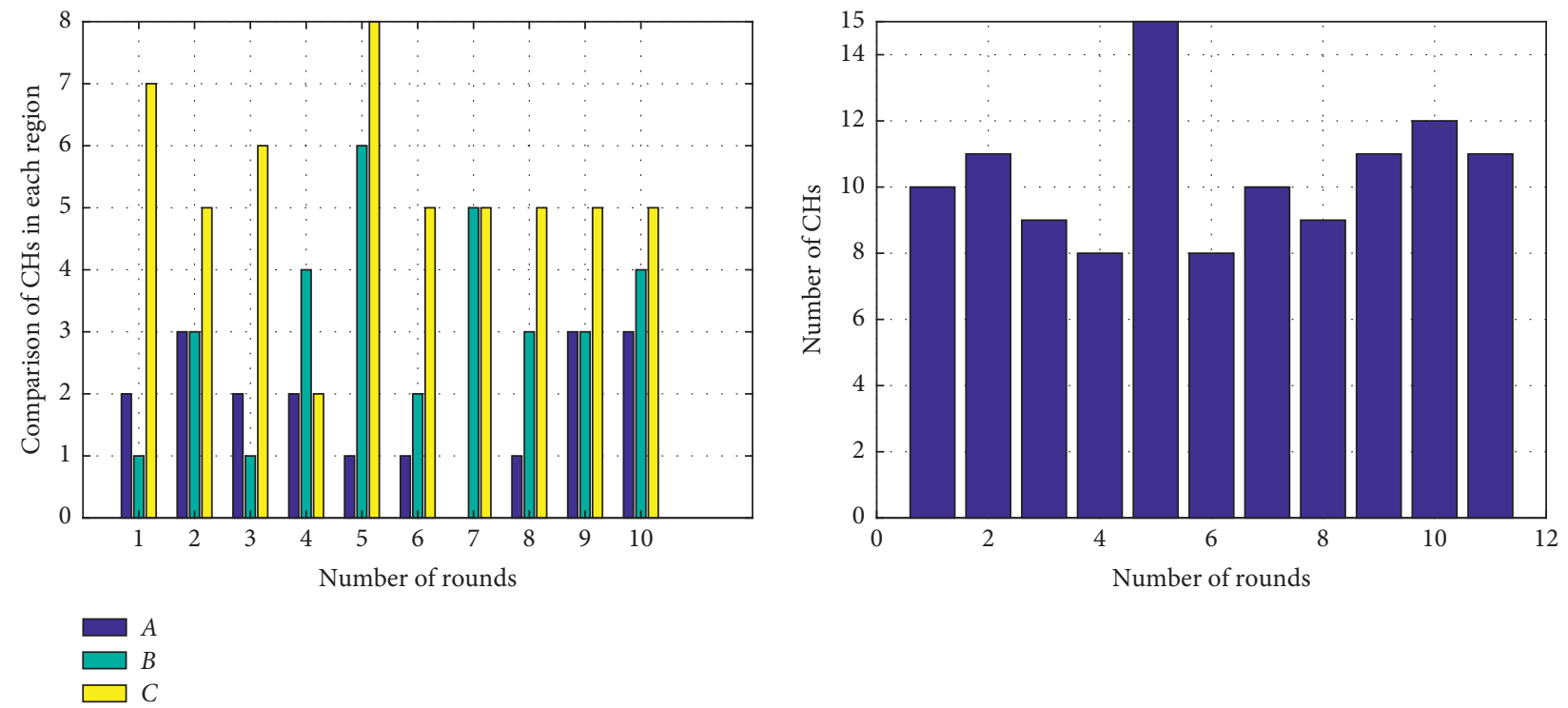

(a)

(b)

FIGURE 16: Continued. 


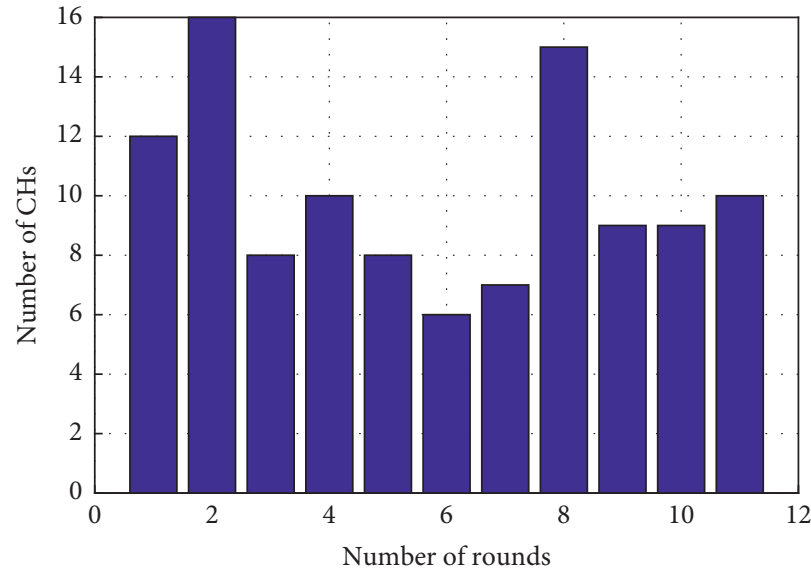

(c)

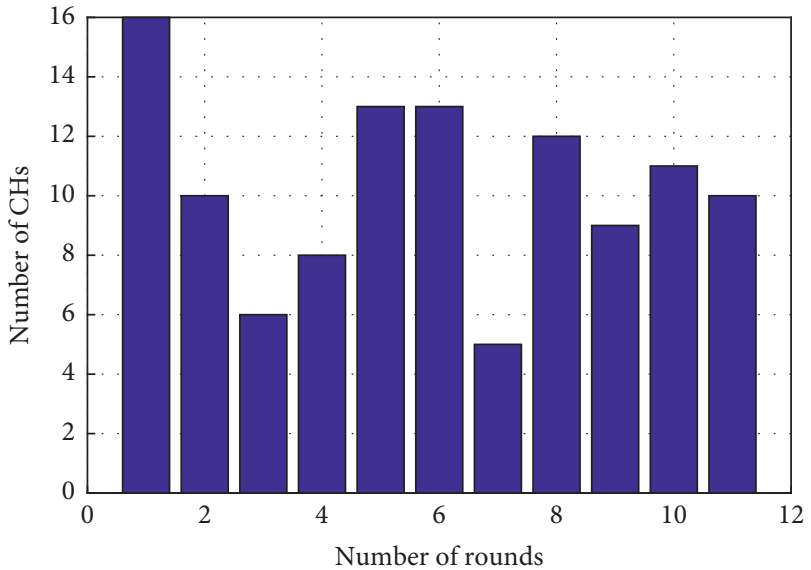

(d)

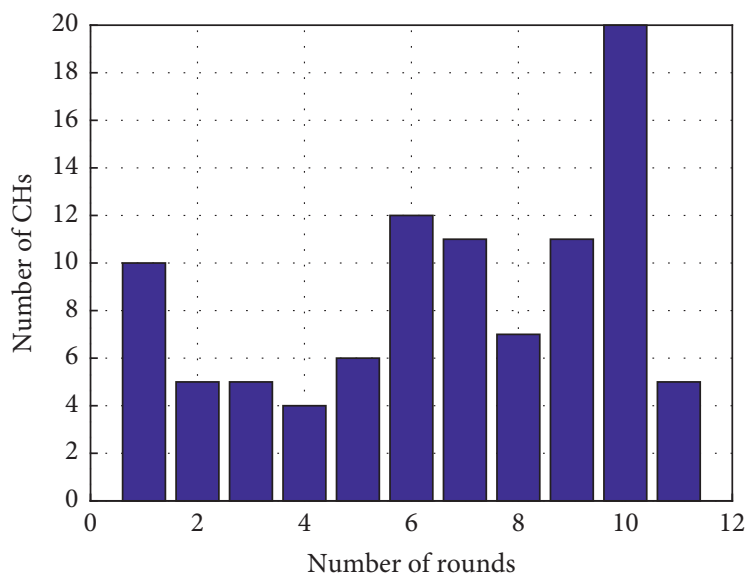

(e)

Figure 16: Statistics of CHs (a) in each zone of FOI-LEACH protocol, (b) of FOI-LEACH protocol, (c) of LEACH protocol, (d) of SEP protocol, and (e) of PECRP protocol with power supply.

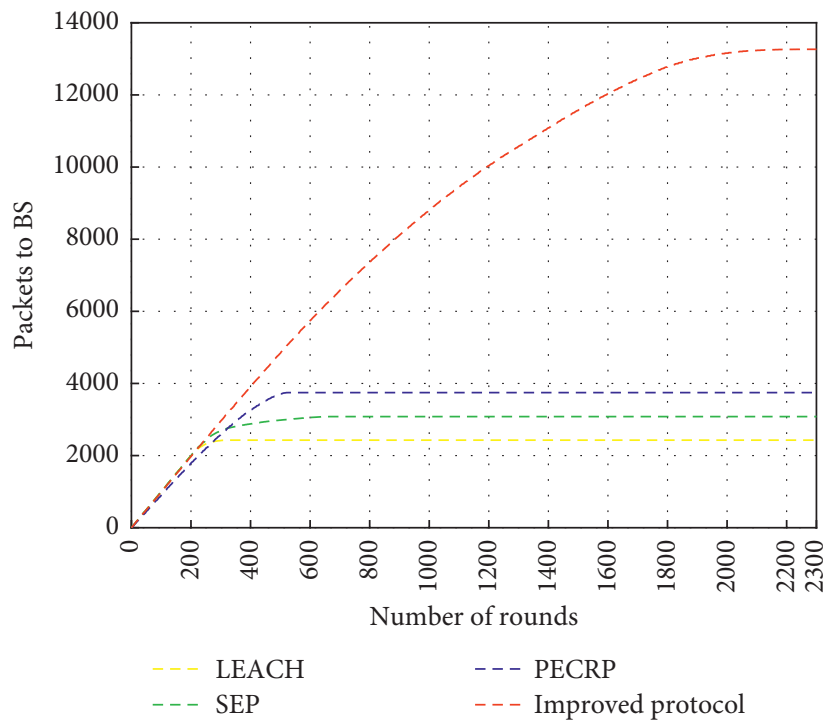

FIgURE 17: Comparison of packets sent to BS from CHs with power supply. 


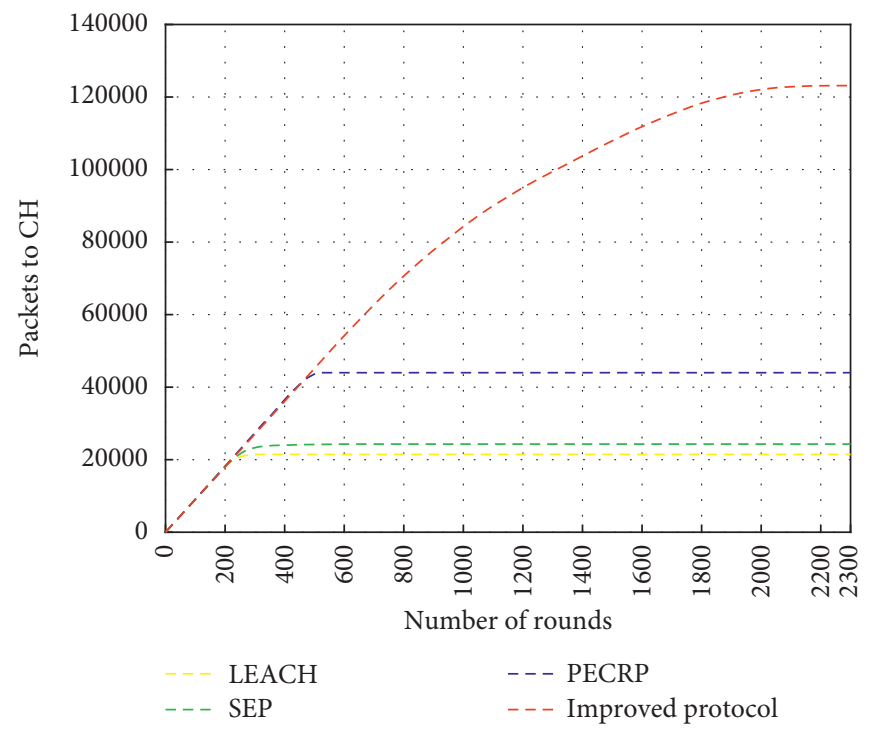

FIGURE 18: Comparison of packets sent to $\mathrm{CHs}$ from nodes with power supply.

node. The supplied energy promotes the extension of the network lifetime and the stability of the networking for the four protocols in varying degrees. For the LEACH protocol, the node began to die at round 177, SEP protocol at round 185, PECRP protocol at round 392, and round 555 for the improved FOI-LEACH protocol.

The distributions of the number of clusters in the four protocols with energy supply simulated for 10 rounds of random elections are shown in Figure 16. Like the previous figures, Figure 16(a) represents the statistics number of $\mathrm{CHs}$ of each zone of FOI-LEACH protocol. Figures 16(b)-16(e) represent the statistics number of $\mathrm{CHs}$ of FOI-LEACH protocol, LEACH protocol, SEP protocol, and PECRP protocol, respectively. Due to the efficient schemes of $\mathrm{CHs}$ electing and clustering, the FOI-LEACH protocol shows significantly better performance than the other three protocols.

(4) Network Throughput. In the environment of self-energy, as shown in Figure 17, the total number of packets sent to BS of the LEACH protocol, SEP protocol, PECRP protocol, and the proposed FOI-LEACH protocol is approximately $2428,3080,3746$, and 13265, respectively. Moreover, in Figure 18, the total number of packages sent to $\mathrm{CHs}$ of the LEACH protocol, SEP protocol, PECRP protocol, and FOI-LEACH protocol is approximately 21400, 24300, 44000 , and 123200 , respectively. We can find that the total number of packets sent by our protocol to BS and $\mathrm{CHs}$ is much higher than the other protocols, which also demonstrated that the proposed protocol has a better network throughout.

\section{Conclusions}

According to the characteristics of field observation instrument networking, an improved protocol FOI-LEACH based on LEACH protocol was proposed in this paper. By considering the energy factor in the $\mathrm{CH}$ election threshold and referring to the SEP protocol to set up two kinds of nodes with different initial energies which are called advanced nodes and normal nodes, the network model of field observation instruments has been established. In addition, to solve the problem that the LEACH protocol consumes much energy during transmission and results in uneven energy consumption for the direct and longdistance transmission, the improved protocol adopted the 3 zones of partitioning mechanism and the multihop transmission mechanism to alleviate and avoid the "hot spot" problems. Furthermore, energy supplies were added to the nodes in $\mathrm{A}$ and $\mathrm{B}$ zones that are responsible for more energy transmission, so as to balance the energy consumption of the network and prolong the network lifetime. In the simulation experiments, we compared the performance of the FOI-LEACH protocol with the LEACH, SEP, and PECRP protocols in two cases, with or without energy supply. Comparing performance indicators of network lifetime, energy consumption rate, stability, and network throughput, the proposed FOILEACH protocol can slow down the death of nodes and the energy consumption rate obviously and shows a significantly better performance than other three protocols. The experimental results demonstrated that the FOILEACH protocol could balance network energy consumption and alleviate the "hot spot" problem to extend the lifetime of network nodes in the field observation instruments network. The future work is to implement and port the improved protocol to the hardware of networking node we designed and developed previously and test and verify the feasibility and stability of the protocol in the actual situation.

\section{Data Availability}

The initial nodes are generated randomly in the network, and there are no observation data used in the simulation experiments. 


\section{Conflicts of Interest}

The authors declare that there are no conflicts of interest.

\section{Acknowledgments}

This work was supported by the National Nature Science Foundation of China (Grant no. 61862038), the Lanzhou Talent Innovation and Entrepreneurship Technology Plan Project (no. 2019-RC-14), and the Foundation of a Hundred Youth Talents Training Program of Lanzhou Jiaotong University.

\section{References}

[1] J. Y. Huo, Z. N. Ren, and Y. R. Yang, A Kind of Instrument Network Node Equipment and Monitoring System, Chinese Patents, Beijing, China, 2017.

[2] J. Y. Huo, Key Technology and Application Research of E-Science Virtual Joint Observation System for Geoscience in Cold and Dry Regions, University of Chinese Academy of Sciences, Beijing, China, 2012.

[3] I. F. Akyildiz, W. L. Weilian Su, Y. Sankarasubramaniam, and E. Cayirci, "A survey on sensor networks," IEEE Communications Magazine, vol. 40, no. 8, pp. 102-114, 2002.

[4] W. R. Heinzelman, A. Chandrakasan, and H. Balakrishnan, "Energy-efficient communication protocol for wireless microsensor networks," in Proceedings of the 33rd Hawaii Intentional Conference on System Sciences, Maui, HI, USA, January 2000.

[5] D. Mehta and S. Saxena, "A comparative analysis of energy efficient hierarchical routing protocols for wireless sensor networks," in Proceedings of the 2018 4th International Conference on Computing Sciences (ICCS), pp. 53-58, Jalandhar, India, August 2018.

[6] C. Karlof and D. Wagner, "Secure routing in wireless sensor networks: attacks and countermeasures," Ad Hoc Networks, vol. 1, 2003.

[7] L. B. Oliveira, A. Ferreira, M. A. Vilaca et al., "SecLEACH-on the security of clustered sensor networks," Signal Processing, vol. 87, 2007.

[8] Y. Y. Gu, G. W. Bai, and J. J. Tao, "AZM-LEACH: autonomous zone-based multi-hop routing protocol in WSNs," Computer Engineering and Applications, vol. 47, no. 20, pp. 58-61, 2011.

[9] L. X. Wang, Research on Routing Technology of Wireless Sensor Networks Based on Data Aggregation, Shenyang Ligong University, Shenyang, China, 2015.

[10] J. P. Shao, Research on Key Energy-Efficient Routing Technology of WSN Based on Cluster, Shenyang Ligong University, Shenyang, China, 2015.

[11] V. K. Arora, V. Sharma, and M. Sachdeva, "A survey on LEACH and other's routing protocols in wireless sensor network," Optik, vol. 127, no. 16, pp. 6590-6600, 2016.

[12] W. B. Heinzelman, A. P. Chandrakasan, and H. Balakrishnan, "An application-specific protocol architecture for wireless microsensor networks," IEEE Transactions on Wireless Communications, vol. 1, no. 4, pp. 660-670, 2002.

[13] S. Al-Sodairi and R. Ouni, "Reliable and energy-efficient multi-hop LEACH-based clustering protocol for wireless sensor networks," Sustainable Computing: Informatics and Systems, vol. 20, pp. 1-13, 2018.

[14] O. Younis and S. Fahmy, "HEED: a hybrid, energy-efficient, distributed clustering approach for ad hoc sensor networks,"
IEEE Transactions on Mobile Computing, vol. 3, no. 4, pp. 366-379, 2004.

[15] B. B. Chen and Y. L. Shi, "WSN routing protocol based on cluster heads selection in regions," Computer Engineering, vol. 37, no. 19, pp. 96-98, 2011.

[16] P. Azada, "Cluster head selection in wireless sensor networks under fuzzy environment," ISRN Sensor Networks, vol. 2013, Article ID 909086, 8 pages, 2013.

[17] S. Soro and W. B. Heinzelman, "Cluster head election techniques for coverage preservation in wireless sensor networks," Ad Hoc Networks, vol. 7, no. 5, pp. 955-972, 2009.

[18] A. Thakkar, "DEAL: distance and energy based advanced leach protocol," in Proceedings of the International Conference on Information and Communication Technology for Intelligent Systems, pp. 370-376, Ahmedabad, India, March 2017.

[19] T. Y. Chang, W. N. Liu, Y. Zhang, and H. Y. Li, "Optimized LEACH protocol based on distance and energy of cluster head," Journal of Hebei University (Natural Science Edition), vol. 39, no. 2, pp. 194-200, 2019.

[20] P. Mehra, M. Doja, and B. Alam, "Stability enhancement in LEACH (SE-LEACH) for homogeneous WSN," EAI Endorsed Transactions on Scalable Information Systems, vol. 6, no. 20, pp. 1-9, 2019.

[21] G. Smaragdakis, I. Matta, and A. Bestavros, "SEP: a stable election protocol for clustered heterogeneous wireless sensor networks," in Proceedings of the Second International Workshop on Sensor and Actor Network Protocols and Applications (SANPA 2004), pp. 1-11, Boston, MA, USA, June 2004.

[22] F. S. Hu and Q. Xiao, "Multi-hop routing algorithm of energybalancing based on LEACH," Journal of Chinese Computer Systems, vol. 35, no. 1, pp. 72-75, 2014.

[23] C. F. Li, M. Ye, G. H. Chen, and J. Wu, "An energy-efficient unequal clustering mechanism for wireless sensor networks," in Proceedings of the IEEE International Conference on Mobile Adhoc and Sensor Systems Conference, p. 604, Washington, DC, USA, November 2005.

[24] E. Alnawafa, I. Marghescu, and MHT, "Multi-hop technique for the improvement of leach protocol," in Proceedings of the 2016 15th RoEduNet Conference: Networking in Education and Research, pp. 1-5, Bucharest, Romania, September 2016.

[25] E. Alnawafa and I. Marghescu, "IMHT: improved MHTLEACH protocol for wireless sensor networks," in Proceedings of the 2017 8th International Conference on Information and Communication Systems (ICICS), pp. 246-251, Irbid, Jordan, April 2017.

[26] J. Y. Huo, Z. Zhang, and C. Tao, "Using IPv6 address advantage to build Network Node of Field observation Instruments in cold and arid areas," China Education Network, no. 5, pp. 45-46, 2018.

[27] L. Mateu and F. Moll, "Review of energy harvesting techniques and applications for microelectronics," in Proceedings of the International Society for Optical Engineering, pp. 359373, Bellingham, WA, USA, October 2005.

[28] G. Wang and R. Y. Wang, "Routing algorithm based on cluster-head optimization for self-energized wireless sensor network," Journal of Computer Applications, vol. 38, no. 334, pp. 201-216, 2018.

[29] X. P. Fan, X. Yang, S. Q. Liu, and Z. H. Qu, "Clustering routing algorithm for wireless sensor networks with power harvesting," Computer Engineering, vol. 39, no. 11, pp. 126$134,2008$. 
[30] C. H. Zhang, "Study on the energy heterogeneous clustering routing protocol for wireless sensor networks," Science \& Technology Vision, no. 13, pp. 71+164-165, 2012.

[31] T. Liu and F. Li, "Power-efficient clustering routing protocol based on applications in wireless sensor network," in Proceedings of the International Conference on Wireless Communications, IEEE Press, Melbourne, Australia, September 2009. 